\title{
Modified Peritoneal Dialysis for Treatment of Acute Renal Failure after Complex Congenital Heart Surgery in Infants
}

\author{
Lefeng Zhang, MD, Yongqiang Jin, MD, Fuqiang Zhang, MD, Hongyin Li, MD, Qingyu Wu, MD \\ Department of Cardiovascular Surgery, First Hospital Affiliated to Tsinghua University, Beijing, China
}

\section{ABSTRACT}

Background: Acute kidney injury (AKI) is one of the common complications in infants and children after complex congenital heart surgery. Peritoneal dialysis (PD) is usually applied for renal replacement therapy (RRT), especially in infants. We investigated the efficacy and safety of modified PD for the treatment of acute renal failure and congestive heart failure after cardiac surgery for congenital heart disease in infants.

Methods: We retrospectively analyzed five consecutive patients from October 2015 to February 2017. The patients were aged from four days to five years old, and all had acute renal failure and congestive heart failure after cardiac surgery. In the five patients treated with modified PD (five males; average weight: $11.2 \pm 5.5 \mathrm{~kg}$ ), we used the Seldinger technique percutaneous abdominal puncture $16 \mathrm{G}$ single lumen central venous catheter instead of the Tenckhoff peritoneal dialysis catheter as a PD catheter. Modified PD was intermittent. We recorded and monitored circulation and metabolism index.

Results: Five cases (100\%) with modified PD were restored to normal renal function. Congestive heart failure was gradually alleviated, and pulmonary and cardiovascular function were improved. Urine volume increased. Neither peritonitis nor catheter leakage occured in any of our cases. Urine volume increased due to PD, from $0.16+0.18 \mathrm{~mL} /$ $\mathrm{kg} * \mathrm{~h}$ before PD to $2.63+1.05 \mathrm{ml} / \mathrm{kg}^{*} \mathrm{~h}$ at the end of PD $(P<.05)$. Serum creatinine, serum urea nitrogen, and serum $\mathrm{K}+$ changed from $85.0 \pm 36.5 \mu \mathrm{mol} / \mathrm{L}, 17.1 \pm 7.5 \mathrm{mmol} / \mathrm{L}$, and $4.57 \pm 0.30 \mathrm{mmol} / \mathrm{L}$ before PD, to $76.0 \pm 36.7 \mu \mathrm{mol} / \mathrm{L}$, $20.1 \pm 11.0 \mathrm{mmol} / \mathrm{L}$, and $4.42 \pm 0.42 \mathrm{mmol} / \mathrm{L}$ at the end of $\mathrm{PD}$, respectively $(P>.05)$. Acidosis, hyperkalemia, hypoxemia and low cardiac output syndrome were improved. All patients were cured and discharged with normal renal function.

Conclusions: We conclude that modified single lumen central venous catheter for PD is a safe, feasible, and less invasive therapeutic strategy for AKI in infants undergoing cardiac surgery, and is worthy of being widely applied in clinical practice.

Received Fanaury 11, 2018; accepted May 1, 2018.

Correspondence: Qingyu Wu, Department of Cardiovascular Surgery, First Hospital Affiliated to Tsinghua University, Beijing, 100016, China; (e-mail: wuqingyu@mail.tsinghua.edu.cn).

\section{BACKGROUND}

Acute kidney injury (AKI) is a serious complication after cardiac surgery, and is associated with increased short-term mortality [Chertow 1998]. Depending on the definition, AKI is encountered in $5 \%$ to $30 \%$ of patients after cardiac surgery. There have been many studies investigating severe AKI, including studies on significant rises in serum creatinine and the need for dialysis [Lassnigg 2004; Wijeysundera 2003; Ryckwaert 2002]. A new classification system for AKI was put forward by the Acute Dialysis Quality Initiative Group in 2004, which was named RIFLE (Risk, Injury, Failure, Loss, and End stage). The RIFLE classification provides a standardized definition of AKI, and has been validated and widely accepted. AKI is divided into three grades (1: RIFLE-R, risk; 2: RIFLE-I, injury; 3: RIFLE-F, failure) according to the changes in serum creatinine compared to the base level. After cardiac surgery, patients had a higher prevalence of RIFLE-defined AKI, which was correlated with short-term mortality [5-7].

Infants have an increased risk of AKI after congenital open heart surgery. The risk factors include ischemia, an acute inflammatory response to extracorporeal circulation, postoperative hypotension, ischemia reperfusion injury, endotoxins and free myoglobin, hemolysis, severe sepsis, acute heart failure, and history of renal disease. According to the criteria of $\mathrm{AKI}$, the reported AKI incidence ranges from $1 \%$ to $17 \%$, and the mortality associated with AKI varies (from 21\% to 70\%) [Jander 2007; Skippen 2005; Ramage 1999].

General treatments for AKI include the correction or removal of risk factors and adjusting capacity. When a patient has volume overload and oliguria or anuria, he or she may need renal replacement therapy (RRT). Peritoneal dialysis $(\mathrm{PD})$, which is simple and low cost, is a preferred technique for infants. Because PD has better hemodynamic stability, it does not require vascular access [Penderson 2008]. The aim of the present study was to evaluate the safety and feasibility of a single lumen central venous catheter, compared to a Tenckhoff peritoneal dialysis catheter for PD in infants with acute renal failure (ARF) after complex congenital heart surgery.

\section{MATERIALS AND METHODS}

\section{Patients}

This retrospective study included five infants undergoing modified single lumen central venous catheter during PD treatment for ARF after complex congenital heart surgery, from October 2015 to February 2017. There were a total of 
Table 1. Clinical Features of the Study Group

\begin{tabular}{lllll}
\hline Patient no. & Sex & age & Weight $(\mathrm{kg})$ & Preoperative diagnosis \\
\hline 1 & M & 5 years & 15.0 & TGA VSD PS \\
2 & M & 4 days & 3.7 & TGA \\
3 & M & 2 years & 12.2 & DORV \\
4 & M & 7 months & 8.0 & TOF \\
5 & M & 3 years & 17.2 & CTGA VSD \\
\hline
\end{tabular}

TGA indicates transposition of great artery; TOF, tetralogy of Fallot; CTGA, corrected transposition of great artery; VSD, ventricular septal defect; DORV, double outlet right ventricle; PS, pulmonary stenosis.

eight patients that needed interventions for the catheters at the same time. Five used single lumen central venous catheters, and the other three used Tenckhoff abdomen catheters. We recorded the indications, results, and complications of PD. If there was history of complex surgical intervention that would hinder catheter placement, we altered the procedural approach to common open surgery to implant the PD catheter. The Ethics Committee of First Hospital Affiliated to Tsinghua University approved this study. The parents of all the patients included in this study signed informed consent forms.

$\mathrm{PD}$ is the preferred treatment for ARF after heart surgery at our center because PD is relatively easy to employ, and severe complications from PD are rare. The indications for the initiation of modified PD in our center include: urine output $<0.5 \mathrm{~mL} / \mathrm{kg} / \mathrm{h}$ for more than four hours; unresponsive to adjustment in fluid therapy, diuretic therapy, or the optimization of vasoactive drugs; postoperative creatinine levels more than double the initial values or more than $115 \mathrm{mmol} / \mathrm{L}$; severe edema and congestive heart failure; potassium > $5.5 \mathrm{mmol} / \mathrm{L}$; severe metabolic acidosis; low cardiac output syndrome; or any combination thereof [Reyhanoglu 2015]. Metabolic acidosis was considered severe if it failed to be corrected after intravenous sodium bicarbonate infusion, adjustment of fluid status, and positive inotropic drugs support. Low cardiac output syndrome is defined as a mean arterial pressure below $40 \mathrm{mmHg}$. Indications for the termination of PD are the recovery of normal urine volume to maintain or achieve negative fluid balance, and normal acid-base status and serum electrolytes.

\section{Modified Peritoneal Dialysis}

A single venous catheter was connected to the dialysis line through a three-way switch, which could penetrate into and eliminate the dialysate. A cardiac surgeon inserted modified PD catheters using the Seldinger technique and local anesthesia. We applied a single lumen central venous catheter (Yixinda ${ }^{\circledR}$, Shenzhen, China), which was used for the administration of intravenous fluids and invasive measurement of the central venous pressure as dialysis catheters, instead of the Tenckhoff PD Catheter (Quinton; Tyco Health Care Group, Mansfield, MA).
The infants were placed in a supine position. The site of puncture was disinfected with antiseptic solution, and a sterile operative area was created across the midline and from the costal margin to the iliac crest. We inserted the single lumen central venous catheter based on predetermined landmarks, which were located through bedside ultrasound. Bedside ultrasound was helpful to identify the empty bladder and the thickness of the abdominal wall, so as to identify the depth of percutaneous puncture needle.

We held a $5 \mathrm{~mL}$ syringe with $16 \mathrm{G}$ trocar connected in one hand, according to the modified Seldinger technique puncture, directed towards the pubic symphysis. We kept the $5 \mathrm{~mL}$ syringe negative pressure slowly forward. When the tip resistance suddenly disappeared and peritoneal effusion emerged into syringe, we immediately stopped the needle and pulled out the steel needle core. Then, the J type guide wire was imported into the $16 \mathrm{G}$ trocar with its navigation devices, and slowly implanted to the Rectovesical pouch or Douglas' Pouch. After the withdrawal of the steel core of the $16 \mathrm{G}$ trocar, a single lumen central venous catheter was inserted along the $\mathrm{J}$ type guide wire about $6 \mathrm{~cm}$ into the abdominal cavity.

Next, we exited the guide wire. We inserted the $16 \mathrm{G}$ trocar slowly, so as to avoid bowel injury. If the drainage of peritoneal effusion was fluent, we circularly sutured the central venous catheter to the skin in order to avoid leakage. The puncture area was then covered by a sterile sticker. The catheter was linked to an airtight system for peritoneal drainage by a two tee switch that was connected separately to the bag of dialysate solutions and the $50 \mathrm{~mL}$ container. Through the use of the $50 \mathrm{~mL}$ container, we could measure the ultrafiltration volume. The two tee switch was used to control movement of the dialysate solution in and out. A sealed sterile container with a capacity scale was placed in a constant temperature electric water bath of 37.0 degrees Celsius.

Peritoneal dialysate volume was started at $10 \mathrm{~mL} / \mathrm{kg}$. The fill time and the drainage time were both 30 minutes. We adjusted the fluid balance by change the dwell time and dextrose concentration. We replaced the dialysate solution bags with new ones every 24 hours.

When the single lumen central venous catheter was obstructed and the drainage of the dialysis fluid was slow, a new catheter of the same type was inserted to take off the blocked catheter. When the patient had cloudy dialysis fluid or fever, we should suspected the patient might have peritonitis. Peritonitis was defined as having leucocytes more than $100 / \mathrm{mm} 3$ (neutrophils $>50 \%$ ), a positive bacterial smear, or culture-positive ascitic fluid. Catheter leakage was defined as visible fluid leakage or wetness around the catheter.

\section{Statistical Analyses}

We used the SPSS version 15 software program (SPSS Inc., Chicago, Illinois, USA) for statistical analyses. The descriptive analyses were expressed as mean \pm standard deviation (SD) for variables with normal distribution, and as median for variables with non-normal distribution. A $P$ value less than .05 was considered statistically significant. 
Table 2. Urea, Creatinine, Urine and Potassium Values of $\mathrm{Pa} \neg$ tients Before and After Peritoneal Dialysis

\begin{tabular}{llll}
\hline & Before PD & After PD & $P$ \\
\hline Urine $\mathrm{mL} / \mathrm{kg} * \mathrm{~h}$ & $0.16 \pm 0.18$ & $2.63 \pm 1.05$ & .005 \\
Creatinine umol/L & $85.0 \pm 36.5$ & $76.0 \pm 36.7$ & .762 \\
Urea $\mathrm{mmol} / \mathrm{L}$ & $17.1 \pm 7.5$ & $20.1 \pm 11.0$ & .294 \\
Potassium mmol/L & $4.57 \pm 0.30$ & $4.42 \pm 0.42$ & .447 \\
\hline
\end{tabular}

PD indicates peritoneal dialysis.

\section{RESULTS}

In this study, we retrospectively analyzed five consecutive patients from October 2015 to February 2017. The patients were aged four days to five years old, and had acute renal failure and congestive heart failure after complex cardiac surgery. Their weights ranged from 3.2 to $15 \mathrm{~kg}(11.2 \pm 5.5 \mathrm{~kg})$. All patients needed inotropic support and mechanical ventilation. They developed acute renal failure and needed PD treatment for RRT.

All five cases $(100 \%)$ with modified peritoneal dialysis were restored to normal renal function. Edema was gradually vanished. Circulation and breathing function were improved, and urine volume increased. Peritonitis and leakage from the catheter did not occur in any of our cases. Urine volume increased from peritoneal dialysis $0.16+0.18 \mathrm{~mL} /$ $\mathrm{kg}^{*} \mathrm{~h}$ before PD to $2.63+1.05 \mathrm{~mL} / \mathrm{kg}^{*} \mathrm{~h}$ at the end of PD $(P<.05)$. Serum creatinine, serum urea nitrogen, and serum $\mathrm{K}+$ changed from $85.0 \pm 36.5 \mu \mathrm{mol} / \mathrm{L}, 17.1 \pm 7.5 \mathrm{mmol} / \mathrm{L}$, and $4.57 \pm 0.30 \mathrm{mmol} / \mathrm{L}$ before $\mathrm{PD}$, to $76.0 \pm 36.7 \mu \mathrm{mol} / \mathrm{L}$, $20.1 \pm 11.0 \mathrm{mmol} / \mathrm{L}$, and $4.42 \pm 0.42 \mathrm{mmol} / \mathrm{L}$ at the end of $\mathrm{PD}$, respectively $(P>.05)$. Acidosis, hyperkalemia, hypoxemia and low cardiac output syndrome were improved. All of the patients were cured and discharged with normal renal function. Patients were followed up every six months. During the follow-up period, all of the patients had normal renal and heart function.

\section{DIscussion}

Acute kidney injury (AKI) is a common complication after heart surgery. AKI increases the morbidity and mortality of the cardiac surgery, and the length of intensive care unit stay time [Mangano 1998; Chertow 1997]. Although the severity of AKI varies, when the patient needs RRT, the mortality rate exceeds $60 \%$ [Swaminathan 2009]. A serum creatinine increase of more than $26.5 \mu \mathrm{mol} / \mathrm{L}$ over preoperation levels is one of the independent predictors of postoperation mortality [Lassnigg 2004].

AKI is the result of interactions between cellular, molecular, metabolic, and hemodynamic factors. Hemodynamic changes that lead to vasoconstriction and renal perfusion reduction are the main pathogeneses of AKI. Despite notable progress in the treatment of AKI, the morbidity and mortality remain high [Burne-Taney 2003]. Because AKI is associated with high rates of morbidity and mortality, RRT has been widely used in order to reduce these rates. The aims of PD are ultrafiltration and the clearance of smaller and larger molecules and metabolites. The dialysis membrane of $\mathrm{PD}$ patients is the peritoneum. Metabolite products from the blood diffuse through the peritoneum into the dialysate, which is drained from abdominal cavity. Metabolites pass through the peritoneum by diffusion and convective motions.

The skin and abdominal muscles at the puncture site should be single purse-string sutured to fix the single lumen central vein catheter and reduce the possibility of leakage. Keeping the central vein catheter free of obstructions is important. The dialysis catheter should be fastened using a sterile sticker to avoid stretching, bending, and obstacle formation. Surgical dressing changes and management of input and output of the dialysate should be strictly aseptic.

The input of the dialysate should be finished in 5-15 minutes to avoid a sudden rise in abdominal pressure, which affects respiration and circulation. The output velocity should be controlled to avoid a sudden decrease of abdominal pressure, which may result in hypotension. It is important to strictly control the temperature of dialysate, as low temperatures can affect the function of the peritoneum. We use a homemade heating system to preheat the dialysate to normal body temperature. The patients need parenteral nutrition and enteral nutrition simultaneously to enhance nutritional support.

With the widespread use of Tenckhoff catheters, the mortality of ARF in infants and young children with congenital heart disease after cardiac surgery has significant decreased [Chan 2003]. Compared with the use of the traditional Tenckhoff PD catheter for PD, the use of Seldinger puncture technology to implant a single lumen central venous catheter for modified peritoneal dialysis is more advantageous. First, the Seldinger puncture technology is less invasive, take less time, and has a smaller impact on circulation. Second, using a single lumen central venous catheter can decrease the surgical risks, such as peritoneal hemorrhage, peritonitis risk, and operative incision infection. Third, the process of pulling off a single lumen central venous catheter is much simpler, helping to avoid secondary surgical damage to open the abdominal cavity. We used gauze to compress the puncture point; if this method were not effective, we would compress the puncture point by suture needle. Fourth, a single lumen central venous catheter implanted by percutaneous puncture has an incision of only about $2 \mathrm{~mm}$, less than a Tenckhoff catheter. Fifth, when the dialysis catheter is blocked, replacing a single lumen central venous catheter is easier. One of the patients had three $16 \mathrm{G}$ single lumen central venous catheter for peritoneal dialysis during 40 days of acute renal failure. The other four infants only used one catheter during the PD.

\section{CONCLUSION}

We conclude that the use of a single lumen central vein catheter as modified PD is a safe, feasible, and less invasive method for ARF in infants after cardiac surgery, and is worthy of being widely applied in clinics. Although modified PD may cause some nonfatal complications, they are uncommon. 
However, modified PD should be used carefully in infants, especially in newborns with ARF. The recommendation of a single lumen central vein catheter as modified PD therapy in infants and older children needs to be verified by further studies with larger patient populations.

\section{REFERENCES}

Arnaoutakis GJ, Bihorac A, Martin TD, et al. 2007. RIFLE criteria for acute kidney injury in aortic arch surgery. J Thorac Cardiovasc Surg 134:1554-61.

Burne-Taney MJ, Rabb H. 2003. The role of adhesion molecules and T cells in ischemic renal injury. Curr Opin Nephrol Hypertens 12:85-90.

Chan KL, Ip P, Chiu CS, et al. 2003. Peritoneal dialysis after surgery for congenital heart disease in infants and young children. Ann Thorac Surg 76:1443-9.

Chertow G, Levy E, Hammermeister K, et al. 1998. Independent association between acute renal failure and mortality following cardiac surgery. Am J Med 104:343-8.

Chertow GM, Lazarus JM, Christiansen CL, et al. 1997. Preoperative renal risk stratification. Circulation. 95:878-84.

Dasta JF, Kane-Gill SL, Durtschi AJ, et al. 2008. Costs and outcomes of acute kidney injury following cardiac surgery. Nephrol Dial Transplant 23:1970-4.

Jander A, Tkaczyk M, Pagowska-Klimek I, et al. 2007. Continuous venovenous hemodiafiltration in children after cardiac surgery. Eur J Cardiothorac Surg 31:1022-8.

Kuitunen A, Vento A, Suojaranta-Ylinen R, et al. 2006. Acute renal failure after cardiac surgery: evaluation of the RIFLE classification. Ann Thorac Surg 81:542.

Lassnigg A, Schmidlin D, Mouhieddine M, et al. 2004. Minimal changes of serum creatinine predict prognosis in patients after cardiothoracic surgery: a prospective cohort study. J Am Soc Nephrol 15:1597-605.

Mangano CM, Diamondstone LS, Ramsay JG, et al. 1998. Renal dysfunction after myocardial revascularization: risk factors, adverse outcomes, and hospital resource utilization. The Multicenter Study of Perioperative Ischemia Research Group. Ann Intern Med 128:194-203.

Pederson KR, Hjortdal VE, Christensen S, et al. 2008. Clinical outcome in children with acute renal failure treated with peritoneal dialysis after surgery for congenital heart disease. Kidney Int Suppl 108:S81-6.

Ramage IJ, Beattle TJ. 1999. Acute renal failure following cardiac surgery. Nephrol Dial Transplant 14:2777.

Reyhanoglu H, Ozcan K, Erturk M, et al. 2015. Renal failure after coronary bypass surgery and the associated risk factors. Heart Surg Forum 18:E6-10.

Ryckwaert F, Boccara G, Frappier JM, et al. 2002. Incidence, risk factors, and prognosis of a moderate increase in plasma creatinine early after cardiac surgery. Crit Care Med 30:1495-8.

Skippen PW, Krahn GE. 2005. Acute renal failure in children un $\neg$ dergoing ardiopulmonary bypass. Crit Care Resusc 7:286-91.

Swaminathan M, Phillips-Bute BG, Patel UD, et al. 2009. Increasing healthcare resource utilization after coronary artery bypass graft surgery in the United States. Circ Cardiovasc Qual Outcomes 2:305-12.

Wijeysundera DN, Rao V, Beattie WS, et al. 2003. Evaluating surrogate measures of renal dysfunction after cardiac surgery. Anesth Analg 96:1265-73. 